\title{
Randomness and Determination, from Physics and Computing towards Biology
}

In Nielsen, M.; Kucera, A.; Bro Miltersen, P.; Palamidessi, C.; Tuma, P.; Valencia, F. (Eds.), SOFSEM 2009: Theory and Practice of Computer Science :Proceedings of the 35th Conference on Current Trends in Theory and Practice of Computer Science, Špindleruv Mlýn, Czech Republic, January 24-30, 2009.

Springer, Series: Lecture Notes in Computer Science, Vol. 5404, Subseries: Theoretical Computer Science and General Issues, 2009, XVII, 670 p, ISBN 978-3-540-95890-1. 


\title{
Randomness and Determination, from Physics and Computing towards Biology
}

\author{
Giuseppe Longo \\ CNRS, Dépt. Informatique - ENS, \\ and CREA, Polytechnique, Paris \\ http://www.di.ens.fr/users/longo
}

\begin{abstract}
In this text we will discuss different forms of randomness in Natural Sciences and present some recent results relating them. In finite processes, randomness differs in various theoretical context, or, to put it otherwise, there is no unifying notion of finite time randomness. In particular, we will introduce, classical (dynamical), quantum and algorithmic randomness. In physics, differing probabilities, as a measure of randomness, evidentiate the differences between the various notions. Yet, asymptotically, one is universal: Martin-Löf randomness provides a clearly defined and robust notion of randomness for infinite sequences of numbers. And this is based on recursion theory, that is the theory of effective computability. As a recurring issue, the question will be raised of what randomenss means in biology, phylogenesis in particular. Finally, hints will be given towards a thesis, relating finite time randomness and time irreversibility in physical processes ${ }^{1}$.
\end{abstract}

\section{Introduction}

In classical physical systems (and by this we mean also relativistic ones) randomness may be defined as 'deterministic unpredictability'. That is, since Poincaré's results and his invention of the geometry of dynamical systems, deterministic systems include various forms of chaotic ones, from weak (mixing) systems to highly sensitive ones to border conditions. Randomness can then be viewed as a property of trajectories within these systems, namely as unpredictability in finite time, [3], [15], [7]. Moreover, ergodicity (à la Birkhoff) provides a relevant and purely mahematical way to define randomness asymptotically, that is for infinite trajectories, still in deterministic systems inspired from physics but independently of finite time predictability of physical processes, [13].

Also recursion theory gave us a proper form of asymptotic randomness, for infinite sequences, in terms of Martin-Löf randomness, [31], [36]. This has been extensively developped by Chaitin, Schnorr, Calude and many others, [10], also in relation to physics.

\footnotetext{
${ }^{1}$ Invited Lecture, 35th International Conference on: "Current Trends in Theory and Practice of Computer Science", Spindleruv mlyn (Czech Republic), January 24-30, 2009. Springer LNCS, 2009.
} 
A third form of randomness must be mentioned: the randomness proper to quantum theories. This randomness is intrinsic to quantum measure and indetermination, two principial issues in quantum mechanics, as, according to the standard interpretation, it cannot be viewed as a form of (hidden or incomplete) determination, [16], [1]. Technically, it differs from classical randomness in view of Bell inequalities and their role in probability measures, [5], [7].

It may be shown that these three forms of randomness differ in finite space and time. Yet, by hinting to some recent results by M. Hoyrup and C. Rojas in the team of the author and by T. Paul, we will see that they merge, asymptotically. This poses several open questions as for the correlations in finite time of classical, quantum and algorithmic radomness, an issue extensively studied by many, as these asymptotic analyses may propose a new perspective.

The infinity of this sequences is essential, as we shall see. Yet, before jumping into infinity, let's see how to randomness differs in the various theoretical frames, at finite time, in reference also to computer networks and concurrency, [4]. Later, we will correlate finite time randomness in different frames, by a conjecture on its relation (equivalence?) to time irreversibility.

Finally, the question will be posed concerning the kind of randomness we may need of in theories of the living state of matter, where complex interactions between different levels of organization, in phylogenesis in particular, seem to give even stronger forms of unpredictability than the ones analyzed by physical or algorithmic theories.

\section{A few structures of physical determination}

In physics, the dynamics and "structures of determination" are very rich and vary from one theory to another (classical, relativistic, quantum, critical state physics...). They propose the theoretical frameworks, the causal relationships (when the notion of causality is meaningful) or, more generally, the correlations between objects or even the objects of a theory themselves. A great principle unifies the various theoretical frameworks: the geodesic principle, a consequence of the symmetries and of the symmetry breakings at the center of all physical theories, [17], [6].

As for computability theory, we are all aware of the new and very relevant role of computing in Natural Sciences. Yet, the reference to computer science in the analysis of natural phenomena is not neutral; it organizes the world by analogy to a formidable conceptual and practical tool, the digital machine, of which the strength resides also (and mainly) in identical iteration. This takes the form of primitive recursion (Herbrand's and Gödel's foundation of computability), which is iteration plus "increment a register". Iteration is at the center of the reliability and portability of the software: it iterates or it does what it is expected to, a thousand or a million times, even in computational environments which differ, logically, a bitï£ ¡but not too much though. Recursion and portability constitute and require iteratability. This is what leads Turing, its inventor, 
to say that his "discrete state machine" is Laplacian", [37] (see also the reflections in [27], [28]). By the analysis of programs, or by iterating computations, its evolution can be predicted. Unpredictability is practical, says he; it is not by principle, whereas it is the interesting principle in the continuous dynamics of the physics of chaotic determinism (Turing's other pioneering exploration, [38]) as well as in quantum mechanics, albeit for other reasons. The situation is radically changing in computer networks and the related theoretical frames for concurrency: the complexity of physical space and time steps in along computations. And randomness pops out.

\section{Randomness}

"Random" is not the opposite of "deterministic", in spite of the opposition of these concepts that is commonly made in computing and biology. As a matter of fact, the analysis of randomness is part of the proposal for a structure of determination of physical processes, in particular in classical dynamics, where randomness is deterministic unpredictability. But it is so also when it is related the very precise and specific notion of quantum indetermination and quantum measure of "deterministic evolutions of the state function" (determined by Schrödinger equation).

\subsection{Classical}

What would a dice say if we were to ask it: "Where will you go?" It would answer: "I will follow a geodesic, an optimal trajectory, from my initial conditions; a course which will minimize the Lagrangian action (energy $\times$ time). My trajectory is perfectly determined by Hamilton's principle, otherwise known as the principle of least action. If you are unable to measure exactly my position-momentum or the boundary conditions, that's your problem: my unpredictability, this randomness you make into a paradigm, is purely epistemic. As a classical object, my evolution is totally deterministic". Now, classical (and relativistic) physical measurement is an interval, by principle (there is at least thermal fluctuation). So the processes which, all the while being deterministic, are "sensitive to the boundary conditions", hence to perturbations or fluctuations below measure, escape prediction, and give us randomness as deterministic unpredictability, within deterministic chaotic systems, [24], [15].

The epistemic nature of classical randomness is also given by the co-existence of two complementary approaches to its analysis. One can understand the properties of dice throwing or coin tossing also by statistics. And probabilities, as measure, may be given a priori on the ground, say, of the symmetries of dice or coins. Thus, the same processes can be analyzed both in terms of deterministic unpredictability and of probabilities or statistical analyses. This a further reason to call classical randomness epistemic, one may easily change perspective.

\footnotetext{
2 That is, (equational or functional) determination implies predictability.
} 
And the phenomenon is rather general. Since Poincaré (1890) we know of the unpredictibility of one of the simplest or dearest deterministic systems: the fragment of the Solar system made out of three celestial bodies in theri gravitational field. On a plane, nine equations, Newton-Laplace style, suffice to deermine it, yet... chaos pops out and today we can quantify its unpredictability, in (astronomically short) finite time, [26]. Of course, it is better to analyse coin tossing in statistical terms (too many equations and rather useless) and the Solar system in equational ones (however,... we could bet on whether the Earth will still be on a "viable" orbit around the Sun in 100 milions years, since this is provably unpredictable, $[26])$. Yet, they belong to the same conceptual frame, as for determination.

\subsection{Quantum}

And if we asked the same question to a quanton, an elementary component of Quantum Physics? Firstly, and this is very important, the quanton must be produced and measured for it to be possible to hear or see its response - it is necessary to prepare the experiment and to choose a measuring instrument. If, for example, we were to throw it towards a Young's double slit and if we were to place an interferometer behind the slits, the quanton would say that it is a wave which will pass through the two slits (hence the interference); if we were to rather place a particle counter behind the slits, it would say that it is a particle which will randomly select which slit to go through (50-50). Funny trajectory... its evolution is indeed determined by a wave equation, Schrödinger's equation, but defined in a Hilbert space, a function space, outside of the world, beyond physical space-time. The measurement which brings us back to space-time in both cases, gives us a 'trajectory' which is not a trajectory, a paradigm of intrinsic randomness which is specific to quantum mechanics, [16], [1].

From a mathematical and experimental standpoint, Bell inequalities and Aspect's experiment $([5])$ demonstrate that this randomness is different than that of dynamical systems: the phenomena of entanglement give way to probability correlations that are incompatible with classical probabilistic "laws". As a matter of fact, if two classical bodies (two coins) interact and then separate in space and time, their evolution is statistically independent. In contrast to this, the result of the probability measures of two quanta, which first interact and then travel away in space, are correlated (this is "quantum entanglement"). In short, if Bob in Paris and Alice in Rome, toss two "entangled quantum coins" (or measure the spin up-down of entangled quantons) at the same (relativistic) moment, they always obtain the same, random, result (or, more generally, the results are correlated). There is no way to act on the result, and thus to transmit instantaneously information, yet... something incompatible with classical (and relativistic) theories happens and this has been extensively checked, by experiments. Moreover, entanglement is the core idea in quantum computing.

In summary, it is fair to call quantum randomness intrinsic or objective, in the sense that randomness is intrinsic to the theory (no ontological commitment is necessary!), [7]. In other words, for the standard interpretation (no 
hidden variables nor hidden determination), the theory is "complete": there is no way to avoid probabilities in measure and entanglement forces non-locality of phenomena as probability correlations. The difference should be clear w.r. to classical randomness, where one can both use a deterministic analysis and an analysis in terms of statistical theories. Once more, in classical physics, in principle, the underlying process are fully determined: there are just non-observable (hidden) fluctuations or perturbations, in border or initial conditions, that may cause massive (observable) changes in finite time (randomness as deterministic unpredictability). Classical physics computes (determines) over approximated measures and produces approximated results. In quantum physics what is determined and computed (by Schrödinger equation), is not what is measured, an exact but random value, whose probability value is the real projection of a complex number (a vector).

\subsection{Computational}

And what would the pixel on your computer screen say, as a discrete image of a physical trajectory? For it to move, there needs to be a program which describes a trajectory starting at this pixel's location. This program could consist in the discretization of the mathematical representation of the most complex (or chaotic, § 3.1) of physical evolutions, that of a turbulence, for instance. Once discretized, this representation (equational, or directly given by an evolution function) affects a discrete database, within a discrete computational environment, made up of pixels, 0s or 1s, quite distinguishable from one another. The natural framework for its geometric description is discrete topology. Within the machine, the measurement of the initial conditions and that made at the boundary will be exact. That is, contrary to the physical (classical) framework with its interval-based metric and topology, the measurement of the initial situation and at the boundary is exact in the case of a digital machine, in the sense that we access digits, one by one. Moreover, in the isolated machine (not part of a network), the measurement (the access to the data) is also absolute, in the absoluteness of its time and space. If the trajectory of your dice, well simulated by means of very realistic images, is reinitialized using the same initial and boundary digital conditions, which can be done exactly, it will be identical, be it twice or a thousand times...

Identical iteration, we insist, is the constitutive principle of the discrete state machine and of its computer programming. There is no randomness in the sequential machine: the pseudo-generators of random sequences are just that, pseudo and, if they are launched again in identical initial conditions (the discrete state machine can do this), they will identically iterate their sequences, well distributed for statistical measurement. They are random in the sole sense of having a "good" distribution of 0s and 1s, without regularities. Randomness is an hardware mistake or, more generally, must be introduced from outside (the theory).

In networks, instead, with the existence of concurrency (different machines, distributed in space, "concurrently" allocated to the same calculation), the situ- 
ation gets more complicated: the space-time of physics, even relativistic, introduces new challenges; in it, there is randomness, even of human or social origin (a computer is switched on/of in the net, by a human). So far, the new randomness which presents itself is manageable and relatively well managed; it is possible to make networks do, in general, what they were programmed to. So, to iterate: network software is globally reliable, portable... owing to the remarkable efforts on the part of computer scientists. The fact that the databases remain discrete, with their well-separated topology, is of course at the basis of these successes. Yet, we believe, the blend of many forms of randomness in concurrent computer networks deserves an ad hoc analysisï£jand major clarification: a plan for future work (see below).

\subsection{Biological}

And if we were to ask a species what will be its fate in the next ecosystem, in one year, in a million years? What "structure of determination" can enable us to speak of this randomness in a rigorous fashion, if randomness there is? And concerning the determination of the phenotype on the basis of the genotype, in ontogenesis? Is it possible to speak about this in the terms of classical physics or should quantum physics be preferred?

A common characteristic in the various forms of physical randomness is due to the predetermination of the spaces of possibilities: random results or trajectories are given among already known possibilities (the six sides of a dice, the spin-up/spin-down of a quanton...). In fact, in quantum physics, even in cases where particles can be "created", sufficiently broad spaces are provided upstream (the Fock spaces of which Hilbert spaces are "foliations"), spaces which capture all the possible states, infinitely many in general. In biology, however, phase or reference spaces (or spaces of possible evolutions) are far from being predetermined. The possible and proper biological observables, phenotypes and species, are not pre-given or there is no way to give them, in a sound theory and in advance. An issue here is that species (and phenotypes) are co-constituted with their environment. To make an analogy with the reasons for chaos in planetry systems, some sort of "resonance effect" takes place in this co-consitutive process. The difference is that in the physical deterministic case, the resonance happens at one (and conceptually simple) level: the gravitational ineractions between a few planets, fully determined by Newton-Laplace equations. In evolution (but also in ontogenesis), the resonance takes place between different levels of organization, each deserving an analysis on terms of an appropiate structure of detemination. That is, between species, individuals, physical landscapes, but also organs and tissues and, very importantly, by two ways interactions between these levels and molecular activities, starting with DNA expression. Moreover, molecular events belong to microphysics, thus possibly subject to quantum analysis, thus, quantum probabilities. By this, one would need a theory encompassing both classical randomness, which may better fit the description of macroscopic interactions, and quantum randomness, as they may be retroacting one on top of the other. 
We are far form having such a theory, even in physics (we will mention a recent merging, but... at infinite time).

While waiting for some sort of "unified" determination and probabilities, in biology and for this epistemic analysis (there is no ontology in the above: we are all made just of molecules), it would be good to have a sort of biological indetermination, comparable to the quantum indetermination of the conjugate dimensions of position/momentum. That is, to analyse the (in-)determination at the level of the passage from one reference space (ecosystem), at a particular moment, to that of the "next" moment. And this passage would "contain" or express the biological (phylogenetic, ontogenetic) trajectories, which are just possibilities in a forthcoming, co-constituted ecosystem. For instance, when, over the course of evolution, a "latent potential" ([22]) manifests in the appearance of a new organ, an highly unpredictable phenomenon, it is the phenotypes and the interaction between individuals which change (the biological observables), and so the whole relevant space of analysis changes. Is this unpredictability to be analysed within a frame of chaotic determination or is it proper indetermination, like quantum randomness? A combination of the two?

Once more, like in quantum physics, there is no ontological commitment here: the reader and the bacteria around/in him/her are all made of molecules and quanta. Yet, the question is: which theory is a good one for dealing with these strange bags of molecules we are? Darwin proposed a very relevant theory of species and organisms, totally disregarding molecules, but looking at the proper observables. Then, one day, perhaps, we will have a unification: we will be able to grasp at once molecule and species. So far, the so called "synthetic theory", which pretends to understand evolution in molecular terms, has been shown to be incomplete: no way to understand the phylogenetic drift in terms of random mutations only, [22], [23]. In short, this is (also) because massive retroactive effects, from the ecosystem to the phenotype down to the genotype intervene even at the molecular level (e.g. mutations under stress) or make the expression even of identical DNA radically differ. A global analysis of some aspects of the phylogentic drift (its complexification as symmetry breaking) may be found in [8], by an analysis of "anti-entropy" as biologically organized lowering of disorder.

I insist on the issue of randomness in biology, as it is amazing to observe that leading biologists, still now and along the lines of Crick and Monod ([32]), contrappose determination and randomness according to Laplace's split: determined means predictable (in general, as Laplace knew that isolated critical points exist, where "des nuances insensibles" may lead to unpredictable trajectories), and radomness is its opposit (non-determination), to be analyzed by statistics and probabilities (to which Laplace greatly contributed). Along these laplacian lines, determinism, as it is predictable, yields "programmable", which leads to the the idea that the "DNA is a program" (see [18] for an history, [30] for a critique from the point of view of physics and Theory of Programming). Yet, since Poincaré (1890), we know that classical randomness is deterministic unpredictability and that unpredictability pops out almost everywhere in non-linear systems. 
In conclusion, determination as necessity in life phenomena, understood in a laplacian way, is far removed from the frameworks of modern determination in physics, classical or quantum, even if it is supplemented by a few speckles of randomness. Crick's "central dogma" and the "one gene - one enzyme" hypothesis in Molecular Biology are good example of this. They guided research for decades and, the first, is still now believed by many, modulo the addition of a few "epigenetic factors" and "norms of reaction" (for an alternative view, see [9]; more discussions and references are in [30]). By their linear causality, these asumptions are the opposite of the views on the interplay of interactions in XXth century physics. In these modern physical frames, causes become interactions and these interactions themselves dynamicaly constitute the fabric of the universe and of their manifestations; reshaping this fabric modifies the interactions, intervening upon the interactions appears to reshape the fabric, [6].

\subsection{More on randomness in computing}

Physics has been able to propose two different notions of randomness in finite time: classical deterministic unpredictability and quantum randomness. As we shall mention, they merge in infinite time. Biology badly needs its own notion, while in search, of course, for unification with physical (molecular?) structures of determination.

Computing generated its own very robust notion for infinite sequence, MartinLöf randomness, our next topic, yet it has no internal notion of randomness at finite time. Following Kolmogorof, Chaitin, Levin, Calude and many others deeply analysed sequence incompressiblity and showed that for infinite sequences, under suitable conditions, the incompressibility of initial segments yields Martin-Löf randomness. But, unless the physical generating process is spelled out, a finite sequence whose length coincides with its shortest generating program, is not random, it is just algorithmically incompressible. In other words, it is pseudorandom in the strongest wayï£jand it is impossible to see in it any regularity what so ever. Yet, if one stays within theory of computation and no physical process is mentioned, there is no other way to give/conceive it but by a program, a formal/linguistic matter, as long as we stay in the finite.

Some may see this as a terminological nuance, yet too much confusion in computing deserves clarification. Consider, say, a so called "non-deterministic" Turing Machine. This is just a formal, deterministic device, associating a set of numbers to a number. Its evolution is determined by an ill-typed input-output function. Indeed, it is a useful device as it allows to speed up computations by a form of basic parallelism. Yet, as long a physical process, chosing, at each step, one or a few of the elements in the output set, is not proposed, one cannot discuss about "determination" nor "randomness": is it classical? quantum? Similarly for a finite sequence. Of course, a physical random sequence is incompressible, in principle, but the converse is false or ill defined.

\footnotetext{
3 "Genetic Information" goes one-way, from DNA to RNA to proteins (and to the phenotype).
} 
Similarly, the very difficult issue of non-determinism in concurrency deserves a closer attention and comparison with physics. As we said, classical (and relativistic) frames are deterministic, from our chaotic planetary system to coin tossing. Often, non determinism in networks is a "do not care" non determinism: the process works anyway, disregarding the specific underlying computational/determination structure (software and hardware). Other notions are known, and all handle the very difficult situation where, somewhat like in biology, various notions of randomness interact in a network (classical randomness, 'humans' whim switching on a computer, as we observed, a quantum experiment guiding a computer action in a physics laboratory...). A large area of research deals with these processes by statistical tools, independently of the different causal structures. This is possible as the issue may be considered epistemic: like in classical systems, dice throwing and planetary systems may be analyzed also in purely statistical terms and by probabilities measures, independently of a fine analysis of determination. Yet, the question remains whether a closer analysis of randomness in concurrency may lead to a better understanding, in particular in relation with the rich structures of determination in physics.

\subsection{Towards infinity: merging with physics}

Dynamical unpredictability is a finite time issue: the mathematical determination of an intended physical process, by a set of equations or by an evolution function, does not allow to predict the process beyond a certain finite amount of time. Today, we can compute the upper bound of predictability for the solar system and a lot more. Of course, as for dice or coins, the beginning of randomness, as unpredictability, is... immediate. Note that this forces to relate pure mathematics (the formal determination) and physical processes by the only form of access we have to them: physical measure. It is approximated measure, a physical (classical) principle, jointly to the (non-linear typically) structure of mathematical determination that produce unpredictability: by looking at the equations, say, one understands that, and sometimes even compute (by using Lyapounov exponents for example) when fluctuations below measure give diverging evolutions.

However, we can stay within the purely mathematical realm and give a notion of randomness just by looking at the mathematical determination. The price to pay is the passage to (actual) infinity: these internal notions of randomness are necessarely asymptotic. Many forms of deterministic chaos allow different abstractions of this kind. A weak one, "mixing systems", is sufficient to define Birkhoff ergodicity. In short, a trajectory (or, even a point generating it) is Birkhoff random when, w.r. to any observable (a continuous function taking values on the points of the trajectory), the temporal mean coincide with the spatial one. More formally: given a dynamical system $(D, T, \mu)$, a point $x$ is (Birkhoff) random (or typical, in the ergodic sense) if, for any observable $f$,

$$
\lim _{n} \frac{1}{n}\left(f(x)+f(T(x))+\ldots+f\left(T^{n}(x)\right)\right)=\int f d \mu
$$


That is, the average value of the observable $f$ along the trajectory $\left\{x, T(x), T^{2}(x)\right.$, $\left.\ldots T^{n}(x), \ldots\right\}$ (its time average) is asymptotically equal to the space average of $f$ (i.e. $\int f d \mu$ ).

As already announced, also computability theory may generate randomness. Algorithmic randomness (Martin-Löf, '65, Chaitin and Schnorr) for infinite sequences was originally defined in Cantor Space $D=: 2^{\mathbb{N}}$ given $\mu$, a measure on $D$, an effective statistical test is an (effective) sequence $\left\{U_{n}\right\}_{n}$, with $\mu\left(U_{n}\right) \leq 2^{n}$. That is, a statistical test is an infinite decreasing sequence of effective open sets in Cantor's space (thus, it is given in recursion theory). By this, one can define $x$ to be ML-random if, for any statistical test $\left\{U_{n}\right\}_{n}, x$ is not in $\cap_{n} U_{n}$ (x passes all tests). In short, algorithmically random means not being contained in any effective intersection or to stay "eventually outside" any effective statistical test (to pass all tests).

Now, by non obvious work, M. Hoyrup and C. Rojas, in their thesis under this author's and S. Galatolo's supervision, have reconstructed a fully general frame for computable dynamics. That is, they have given an effective structure to physically interesting dynamical systmes where Birkhoff ergodicity applies. By this, they could generalize Martin-Löf randomness to these spaces (under the weaker form of Schnorr's randomness) and show that it coincides with ergodicity (Birkhoff randomness) [19], [20].

Next building, in the same institution, T. Paul recently proved that the peculiar role of "infinite time" is somehow strenghtened by the comparison between quantum (intrinsic) indeterminism and classical (chaotic but deterministic) unpredictability [33], [34]. In a very syntethic way, he showed that, at the limit of small values of the Planck constant (semiclassical limit) and related long time behaviour, the two notions merge.

If the analogy is not too audacious, these asymptotic unifications have a wellknown predecessor. Boltzmann "unified" thermodynamics and classical physics, asymptotically. In particular, he derived the second principle of thermodynamics, by an analysis of (or "as if there were") infinitely many particles in a finite volume. That is, the "reduction" is performed at the limit, by the (thermodynamic) integral over infinitely many trajectories. More than a reduction, thus, to classical physics, it is a matter of unification by the invention of a new unifying frame. In short, in order to understand the abstract thermodynamic principles in terms of particles' trajectories, Boltzmann had to redesign classical trajectories in the novel terms of statistical mechanics, he had to assume molecular chaos and perform a limit transition, an extremely original step, far away from Newton's system.

\section{A thesis on randomness and irreversible time}

The 'thesis' I want to hint here (just a thesis so far, to be enriched by proofs or arguments we work at) is that finite time randomness is 'related' to irreversible time, in all the main physico-mathematical contexts. That is, one has irreversible time exactly "in presence" of randomness (and viceversa) in all the theoretical 
frames mentioned below (and above). Of course this thesis, yet to be 'proved', is worth per se, as it commits our understanding of randomness, a not so clearly defined notion in full generality, to irreversibility of time. Let's hint to a few arguments for it.

\subsection{Classical}

In Euler-Lagrange or Newton-Laplace equations, typically, time is reversible. The same should be said, of course, for Einstein equations. Time appears squared, so its sign has no influence on the evolution of the intended systems. But also a linear field (time is not squared) may be reversed: reverse the sign of the field and the particle goes backwords, in space and time. Reversibility corresponds to the mathematical invertibility of the function describing the trajectory.

Poincaré, as recalled above, proved for us that non-linear (field) equations generate chaos, to put in modern terms. Diverging trajectories, whose divergence may be measured in various way (Lyapounov exponents, entropy growth...), imply finite time unpredictability of generic trajectories. That is, arbitrarely close points may rapidely diverge or fluctuations below observability, thus not knowable, lead to observably different (unpredictable) evolutions. This is exactly classical randomness, from dice to the solar system, both determinsitic (see above, and) chaotic systems, and many others. Of course, the finite time of unpredictability is very different in the two cases: from fractions of seconds to milions of years. But unpredictability puts an arrow on time: you need a future w.r. to a present to define it. Mathematically, non-linearity implies non-invertibility of functions and, thus, of the related trajectories: this is another way to impose a direction on time.

\subsection{Thermodynamics}

Thermodynamics is the realm of oriented time: the second principle, for increasing entropy, is the paradigm of time irreversible processes. But, what is entropy growth in general? It is downgrading of energy, by a diffusion process. And each diffusion, in physics, is given by random paths. Again then, irreversibility of time 'goes toghether' with randomness. Note that the divergence of trajectories in the non-linear dynamics mentioned above may also be measured in terms of proper notions of entropy growth.

\subsection{Quantum}

Schrödinger equation is linear, yet it is 'reversible', its own way, by the peculiar role of the imaginary ' $i$ ': the inversion of time, $-t$, yields a $-i$ whose behaviour, squared, is the same as that of $i$. Yet, what is computed, the evolution of the state function by Schrödinger equation, is not what is measured. And measure, in quantum mechanics is a highly time irreversible process: no way to reconstruct the proper complex values after their projection over the real numbers 
(their modules) is given. Observe finally, that measure is a probability value of a random process. Once more, than, randomness pops out exactly when one deals with a time irreversible process.

How can algorithmic randomness help in this? As hinted above, the (algorithmic) notion of randomness for finite strings (that is, generated in finite time, in principle) is purely formal: it is just a (very relevant) matter of algorithmic uncompressibility. The sentence, in theories of algorithms, "given a random finite sequence", has no contextual meaning, as for the analysis of physico-mathematical determination, unless a physical agent producing the sequence is provided: will it be a series of quantum measure? or a series of coin tossing? the result of networks activity? their randomness differ, as we know.

Yet, computability theory provides a very robust, infinite time, notion of randomness, which may be unified (asymptotically) to the two forms of physical randomness mentioned above, both given also in finite time.

Does this prove that "all physical processes are computable" (an extended Church Thesis), at least as for classical ones? Quite the contrary. Algorithmic randomness is a form of (strong) undecidability (non recursive enumerability) of infinite sequences, as an algorithmic random sequence has no infinite recursive enumerable (sub-)sequence.

Thus, the algorithms/physics correspondence above proves an equivalence of the negations, in deterministic systems: unpredictable is (asymptotically) equivalent to (strongly) non effective. Thus, algorithmically effective processes correspond, as for the structure of determination, only to physical deterministic and predictable ones, a few rare processes in Nature (a simple pendulum, a falling stone... with negligible friction).

These digital devices that are changing the world, are a rather extraodinary invention of ours, comparable to and as important as the invention of the alphabet, an early far from neutral discretization of language and knowledge, [25]; in particular, the sequential machines are alpha-numeric discrete state divices of incredible logical and physical stability. And, as for their use in the simulation of all sorts of physical processes, Turing's insightful distinction between "model" and "imitation" helps to understand their effectiveness and pertinence w.r. to intelligibility of natural processes ([37], [27], [28]).

In [29] further elements are given concerning these final remarks, including an analysis of the limits of the (classical) computational language in (quantum) physics. The point is not "super-computing", that is the search for physical processes which would compute non-Turing-computable functions, but the varying meaning of equational and functional approaches in very different contexts. Quantum computing, typically, requires a truely novel insigth.

\section{References}

1. Anandan J. Causality, "Symmetries and Quantum Mechanics". Foundations of Physics Letters, vol.15, no. 5, 415 438, October, 2002. 
2. Adler R. L. Topological entropy and equivalence of dynamical systems, American Mathematical Society, 1979.

3. Alligood K., Sauer T., Yorke J., Chaos: an introduction to Dynamical Systems, Springer, New York, 2000.

4. Aceto L., Longo G., Victor B. (eds.) The difference between Sequential and Concurrent Computations. Special issue, Mathematical Structures in Computer Science, Cambridge U. Press, n. 4-5, 2003.

5. Aspect A., Grangier P. and Roger G., "Experimental Realization of the EinsteinPodolsky-Rosen-Bohm Gedankenexperiment: A New Violation of Bell's Inequalities", Phys. Rev. Let. 49, p.91, 1982.

6. Bailly F., Longo G., Mathématiques et sciences de la nature. La singularité physique du vivant. Hermann, Paris, 2006 (English introduction, downloadable; ongoing translation).

7. Bailly F., Longo G. "Randomness and Determination in the interplay between the Continuum and the Discrete", Mathematical Structures in Computer Science, vol. 17, n. 2, 2007.

8. Bailly F., Longo G. "Biological Organization and Anti-Entropy", to appear in Journal of Biological Systems, 2008.

9. Brett D., Pospisil H., Valcárcel J., Reich L., Bork P. "Alternative splicing and genome complexity". Nature Genetics 30, 2001.

10. Calude C. Information and Randomness: An Algorithmic Perspective. Springer-Verlag New York, 1994.

11. Calude C., Stay M. "From Heisemberg to Gödel via Chaitin", International J. Theor. Phys. 44 (7), 2005.

12. Connes A., A. Non-commutative Geometry, Academic Press, 1994.

13. Cornfeld I., Fomin S. and Sinai Ya. G., Ergodic Theory. New York: SpringerVerlag, 1982.

14. Dahan Delmedico A., Chabert J.-L., Chemla K. Chaos et déterminisme, Seuil, 1992.

15. Devaney R. L., An introduction to Chaotic Dynamical Systems, AddisonWesley, 1989.

16. Feynman R., Lectures in Physics. Addison-Wesley, 1966.

17. van Frassen B., Lois et symetries, Vrin, Paris, 1994.

18. Fox Keller E., The Century of the Gene, Gallimard, 2000.

19. Galatolo S., Hoyrup M. and Rojas C., "Effective symbolic dynamics, random points, statistical behavior, complexity and entropy", submitted, 2008.

20. Galatolo S., Hoyrup M. and Rojas C., "A Constructive Borel-Cantelli lemma. Constructing orbits with required statistical properties", submitted, 2008

21. Gandy R., "Church's Thesis and the principles for Mechanisms" in (Barwise et al. eds) The Kleene Symposium, North Holland, 1980.

22. Gould S. J. Wonderful Life, WW. Norton, 1989.

23. Lecointre G., Le Guyader H., Classification phylogénétique du vivant, Paris, Belin 2001.

24. Lighthill J. The recent recognized failure of predictability in Newtonian dynamics, Proc. R. Soc. Lond. A 407, 35-50, 1986.

25. Herrenschmidt C. Les trois écritures, Gallimard, 2007.

26. Laskar J., "Large scale chaos in the Solar System", Astron. Astrophysics, 287, L9 L12, 1994.

27. Longo G., "Laplace, Turing and the 'imitation game' impossible geometry: randomness, determinism and programs in Turing's test". In Epstein, R., Roberts, G., 
\& Beber, G. (Eds.). The Turing Test Sourcebook. Dordrecht, The Netherlands: Kluwer, 2007.

28. Longo G. "Critique of Computational Reason in the Natural Sciences", In Fundamental Concepts in Computer Science (E. Gelenbe and J.-P. Kahane, eds.), Imperial College Press/World Scientific, 2008.

29. Longo G., Paul T., "The Mathematics of Computing between Logic and Physics". Invited paper, Computability in Context: Computation and Logic in the Real World, (Cooper, Sorbi eds) Imperial College Press/World Scientific, 2008.

30. Longo G., Tendero P.-E., "The differential method and the causal incompleteness of Programming Theory in Molecular Biology". In Foundations of Science, n. 12, pp. 337-366, 2007 (preliminary version in French in Evolution des concepts fondateurs de la biologie du XXIe siècle, DeBoeck, Paris, 2007).

31. Martin-Loef, P. "The definition of random sequences". Information and Control 9: 602-619, 1966.

32. Monod J. Le Hasard et la Nécessité, PUF, 1973.

33. T. Paul, "Échelles de temps pour l'évolution quantique à petite constante de Planck", Séminaire X-EDP, École Polytechnique, Palaiseau, 2008.

34. T. Paul, "Semiclassical analysis and sensitivity to initial conditions", Information and Computation, to appear, 2008a.

35. Pour-El M.B., Richards J.I., Computability in analysis and physics. Perspectives in mathematical logic, Springer, Berlin, 1989.

36. Rojas C. "Computability and Information in models of Randomness and Chaos", Math. Struct. in Computer Science, vol. 18, pp 291-307, 2008.

37. Turing A.M., "Computing Machines and Intelligence", Mind, vol. LIX, n. 236, p. 433-460, 1950.

38. Turing A.M., "The Chemical Basis of Morphogenesis", Philo. Trans. Royal Soc., vol. B237, p. 37-72, 1952.

39. V'yugin, Vladimir V., "Ergodic Theorems for Individual Random Sequences", Theoretical Computer Science, vol. 207, p.343-361, 1998. 